\title{
DISPONIBILIDADE DE HORAS DE FRIO NA REGIÃO CENTRAL DO RIO GRANDE DO SUL: 2 - DISTRIBUIÇÃO GEOGRÁFICA
}

\author{
CHILLING HOURS IN THE CENTRAL REGION OF RIO GRANDE DO SUL STATE: \\ 2 - GEOGRAPHIC DISTRIBUTION
}

\section{Galileo Adeli Buriol $^{1}$ Arno Bernardo Heldwein ${ }^{1}$ Flávio Miguel Schneider ${ }^{2}$ Valduino Estefanel $^{2}$ Helenir Trindade de Oliveira ${ }^{3}$ Marino Antonio Didoné ${ }^{4}$.}

RESUMO

Foram traçadas as cartas da distribuição de disponibilidade de horas de frio na região Central do Rio Grande do Sul. Para tal, utilizaram-se os dados de disponibilidade de ocorrência do número médio acumulado de horas de frio $<13^{\circ} \mathrm{C} e$ $<7^{\circ} \mathrm{C}$ nos meses de abril a setembro de dez estações meteorológicas localizadas na região. Traçaram-se as isolinhas de diferentes totais de valores médios acumulados do número de horas de frio para a probabilidade de ocorrência de $50 \%$. Para auxiliar no traçado das isolinhas foram utilizadas equações de regressão, estimando os valores do número de horas de frio em função da altitude e latitude. Os valores mais baixos do número de horas de frio ocorrem nas áreas de menor altitude, como nos vales dos rios Jacuí, Vacacaí e Ibicuí e afluentes, e os valores mais elevados nas áreas de maior altitude como nas partes do Planalto e Serra do Sudeste.

Palavras-chave: cartas climáticas, horas de frio, frutíferas caducifóleas.

\section{SUMMARY}

Maps of chilling hours distribution in the central region of Rio Grande do Sul, Brazil, were drawn. Mean of chilling hours bellow $7^{\circ} \mathrm{C} \quad(\mathrm{HF}<7)$ and $13^{\circ} \mathrm{C} \quad(\mathrm{HF}<13)$ accumulating every 15 days up to certain date were used. Isolines of different intensities were drawn using average accumulated values of the number of chilling hours for the occurrence probability of $50 \%$. Two distribution combinations were used to determine the occurrence probability of chilling hours. One was a discrete distribution to verify if there was chilling hours accumulated during the 15 day interval and the other one was a continuous distribution used to identify the accumulate chilling hours. Regression equations that adjusted chilling hours values as a function of the local latitude and altitude were used to simplify isolines drawing. Lower values of accumulate chilling hours below $7^{\circ} \mathrm{C}$ and below $13^{\circ} \mathrm{C}$ were observed in locals with low altitudes, such as Jacuí, Vacacaí and Ibicuí river valleys and main tributary streams. However, higher values were observed in high altitudes mainly in Serra do Sudeste and Planalto highland areas.

Key words: chilling hours, deciduous fruit tree, regional distribution maps.

\section{INTRODUÇÃO}

A disponibilidade de frio invernal é um dos principais fatores que condicionam o desenvolvimento e a produtividade das frutíferas criófilas. O frio invernal é quantificado pela variável "horas de frio" que é o tempo, em horas, em que a temperatura do ar permanece abaixo da temperatura base superior para quebra de dormência. Além da diminuição da produção, a insuficiência em frio invernal afeta também a qualidade dos frutos e diminui o vigor e a longevidade das plantas (CHANDLER et al., 1937; LEDESMA, 1950; PETRI \& PASQUAL, 1982). Dessa forma, a quantificação das horas de frio local e/ou regional é muito importante e necessária no planejamento da agropecuária.

As horas de frio determinadas para cada estação meteorológica, apresentadas em forma de tabela, possuem a vantagem da precisão, entretanto, em função de serem pontuais, em regiões onde ocor-

\footnotetext{
${ }^{1}$ Engenheiro Agrônomo, Professor Titular, Doutor, Departamento de Fitotecnia, Centro de Ciências Rurais (CCR), Universidade Federal de Santa Maria (UFSM), 97105-900, Santa Maria, RS, Brasil. E-mail: heldwein@ @cr.ufsm.br, bolsista do CNPq. Autores para correspondência.

${ }^{2}$ Engenheiro Agrônomo, Professor Titular, DF, CCR, UFSM.

${ }^{3}$ Bacharel em Geografia, $8^{\circ}$ Distrito de Meteorologia, INMET, MAA.

${ }^{4}$ Aluno do Curso de Agronomia, CCR, UFSM. Bolsista da FAPERGS
} 
rem diferenças significativas no relevo, a sua representação geográfica fica prejudicada. Nesses casos, as cartas climáticas desempenham uma função muito importante, pois possibilitam a delimitação das áreas com diferente disponibilidade de frio em função dos fatores geográficos. A região Central do Rio Grande do Sul enquadra-se nessa situação, pois caracterizase por apresentar predominância de relevo ondulado e acidentado e altitudes variáveis de aproximadamente $60 \mathrm{~m}$ a $550 \mathrm{~m}$, o que determina a existência de diferentes microclimas.

Os zoneamentos agroclimáticos para frutíferas criófilas elaborados para o Estado do Rio Grande do Sul (MOTA et al., 1974) ou de horas de frio (INSTITUTO DE PESQUISAS AGRONÔMICAS, 1989) não apresentam este detalhamento e consideram que a região Central do Rio Grande do Sul é praticamente homogênea. Além disso, MOTA et al. (1974) incluem a parte de menor altitude dessa região como não apresentando horas de frio.

Para a região Central do Rio Grande do Sul, HELDWEIN (1994) e HELDWEIN et al. (2000) determinaram o valor médio, a variabilidade e a probabilidade de ocorrência do número de horas de frio acumulados para cada quinzena de cada mês, utilizando os dados de dez e de nove locais, respectivamente. Para cada um desses locais, foram determinados os valores acumulados de horas de frio com temperatura menor do que $7^{\circ} \mathrm{C}(\mathrm{HF}<7)$ e menor do que $13^{\circ} \mathrm{C}(\mathrm{HF}<13)$, no período de $1^{\circ}$ de abril a 31 de outubro, em intervalos quinzenais. Dessa forma, com esses dados, é possível confeccionar cartas climáticas com diferentes limites de número de horas de frio para a região.

O presente trabalho objetivou, através do traçado das isolinhas do número de horas de frio, mapear esse índice bioclimático na região Central do Estado do Rio Grande do Sul.

\section{MATERIAL E MÉTODOS}

Foram utilizados os dados de probabilidade de ocorrência do número médio acumulado de horas de frio $(\mathrm{HF}<7$ e $\mathrm{HF}<13)$ dos meses de abril a setembro de dez estações meteorológicas, localizadas na região Central do Estado do Rio Grande do Sul: Cacequi, Cachoeira do Sul, Julio de Castilhos, Santa Maria (três locais: cidade, UFSM e Pau Fincado), São Gabriel (Estação do Oitavo Distrito de Meteorologia e Estação da Secretaria da Agricultura e Abastecimento do Rio Grande do Sul), São Sepé e Sobradinho (HELDWEIN et al., 2000). Traçaram-se as isolinhas de diferentes disponibilidades dos valores médios acumulados do número de horas de frio para a probabilidade de ocorrência de $50 \%$. Foram utilizados somente os dados de $50 \%$ de probabilidade por estes representarem o valor médio e, ainda, para evitar a publicação de um número excessivo de mapas. Para auxiliar no traçado das isolinhas, foram utilizadas equações de regressão, estimando os valores do número de horas de frio em função da altitude e latitude. Os valores do coeficiente de regressão $\left(\mathrm{r}^{2}\right)$ não foram muito elevados, embora fossem significativos a $1 \%$ de probabilidade.

Como base cartográfica, utilizou-se o mapa hipsométrico do Estado do Rio Grande do Sul, publicado pelo Conselho Nacional de Geografia/Instituto Gaúcho de Reforma Agrária, escala 1:750.000, considerando-se a parte compreendida entre as latitudes de $29^{\circ} 00^{\prime} \mathrm{S}$ a $30^{\circ} 30^{\prime} \mathrm{S}$ e as longitudes de $52^{\circ} 30^{\prime} \mathrm{W}$ a $54^{\circ} 30^{\prime} \mathrm{W}$.

\section{RESULTADOS E DISCUSSÃO}

Na figura 1, é apresentada a distribuição geográfica do número médio de horas de frio, abaixo de $7^{\circ} \mathrm{C}$, na região central do Rio Grande do Sul. A representação cartográfica da disponibilidade de horas de frio para o mês de abril não foi realizada devido ao valor ser insignificante. No mês de maio, na maior parte da região, os valores não ultrapassam a 50 horas, e os mais elevados, de 50 a 100 horas, ocorrem somente nas partes de maior altitude, ao norte, no Planalto e ao sul, na Serra do Sudeste. Nos meses subseqüentes ao mês de maio, a distribuição espacial da soma acumulada do número de horas de frio inferior a $7^{\circ} \mathrm{C}$, para cada mês, apresenta a mesma tendência: os menores valores, ocorrendo nas partes de menor altitude, como no vale dos rios Jacuí, Vacacaí, Ibicuí e seus afluentes, e os valores mais elevados, nas partes de maior altitude, como no Planalto e na Serra do Sudeste. Até o final de setembro, ocorre, em média, um número acumulado de horas de frio que vai de 100-200, nas áreas de menor altitude, a 500-600 horas, nas áreas mais elevadas. Isso comprova a grande heterogeneidade da orografia da região, condicionando de forma significativa a variação espacial do número de horas de frio.

Com esses resultados, verifica-se que, na região, encontram-se áreas com um número médio anual acumulado de horas de frio superior àquele obtido por MOTA $\boldsymbol{e}$ t al. (1974) e INSTITUTO DE PESQUISAS AGRONÔMICAS (1989). Para estes, as áreas com valor médio mais elevado na região em estudo, apresentam de 300-400 horas. A maior discordância ocorre com os valores mencionados no trabalho de MOTA et al. (1974), o qual apresenta a parte central de região climática da Depressão Central com zero horas de frio como valor normal. 


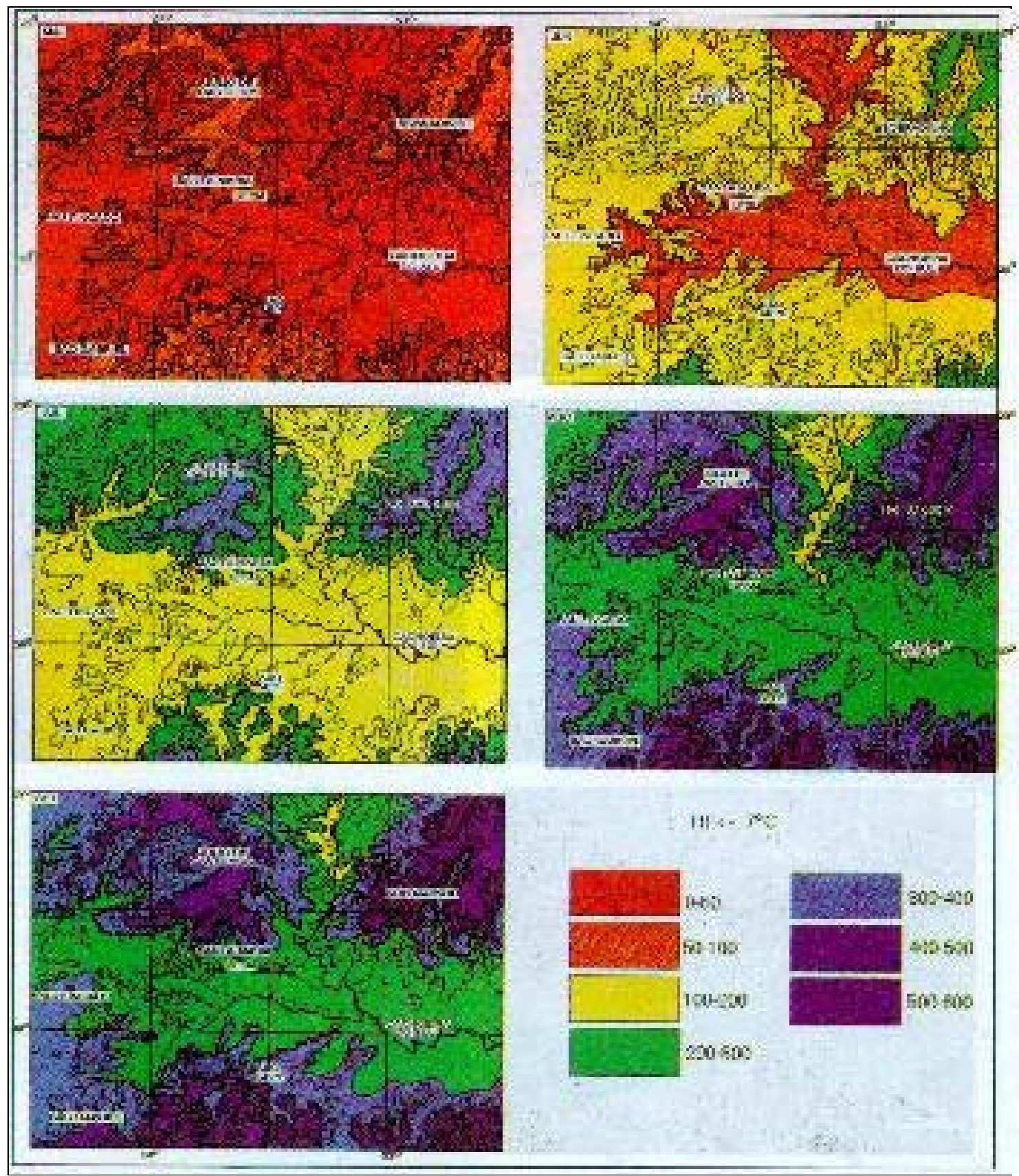

Figura 1 - Número de horas acumuladas com temperatura inferior a $7^{\circ} \mathrm{C}$ para a região central do Estado do Rio Grande do Sul, nos meses de maio, junho, julho, agosto e setembro.

Na figura 2, observam-se as áreas com diferentes somas, em número de horas, de temperaturas abaixo de $13^{\circ} \mathrm{C}$. Para o mês de maio, a distribuição é semelhante àquela das horas de frio abaixo de $7^{\circ} \mathrm{C}$ : os valores mais baixos, 200 a 300 horas, ocorrendo nas partes de menor altitude, como no vale dos rios
Jacuí, Vacacaí, Ibicuí e afluentes, e os valores mais elevados, nas partes de maior altitude, como no Planalto e Serra do Sudeste.

Os valores referentes aos meses de junho, julho, agosto e setembro, considerando a soma acumulada do número de horas de frio para cada mês, 

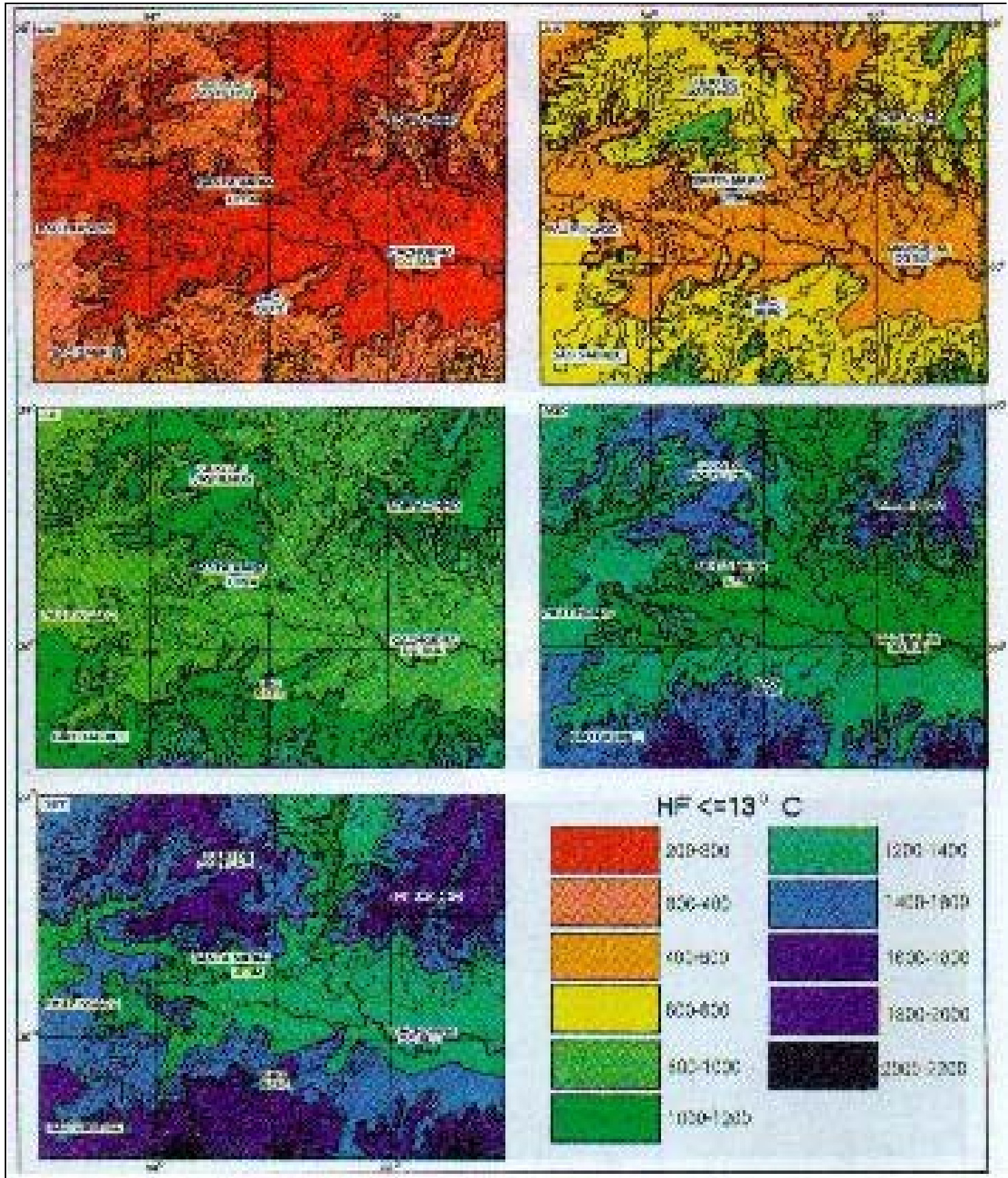

Figura 2 - Número de horas acumuladas com temperatura inferior a $13^{\circ} \mathrm{C}$ para a região central do Estado do Rio Grande do Sul, nos mese de maio, junho, julho, agosto e setembro.

apresentam distribuição geográfica semelhante àquela do mês de maio. Os valores médios para todo o período de $1^{\circ}$ de maio a 30 de setembro variam de 1100-1200, nas áreas de menor altitude, a 20002200 , nas áreas de maior altitude.
A soma acumulada dos valores médios do número de horas de frio e sua distribuição geográfica na região indicam que as áreas de baixas e médias altitudes, como a Depressão Central e os vales dos rios Jacuí, Vacacaí, Ibicuí e seus afluentes devem ser 
cultivadas somente com espécies frutíferas que possuem baixa exigência em frio, como pessegueiros e nectarinas de ciclo precoce e médio, amoreira preta, caquizeiro, algumas nogueiras, figueira e algumas cultivares de videiras. Nas áreas de maiores altitudes, situadas no Planalto e na Serra do Sudeste, poderão ser cultivadas com espécies frutíferas mais exigentes em frio, como pessegueiros de ciclo tardio, ameixeiras, videiras, kiwi e nogueiras. A região não apresenta áreas com disponibilidade de frio para frutíferas muito exigentes em frio, como a macieira e a pereira.

\section{CONCLUSÕES}

Na região Central do Rio Grande do Sul, ocorre variação espacial significativa de horas de frio associada principalmente com a altitude.

As disponibilidades de frio invernal ocorrentes na região Central do Rio Grande do Sul permitem recomendar o cultivo de frutíferas com apenas baixas e médias exigências em frio, sendo as últimas nas áreas de maior altitude.

\section{AGRADECIMENTOS}

Os autores agradecem ao Oitavo Distrito de Meteorologia - INMET, ao Departamento de Portos, Rios e Canais e à
Secretaria da Agricultura e Abastecimento do Rio Grande do Sul, pela permissão de utilização dos dados meteorológicos.

\section{REFERÊNCIAS BIBLIOGRÁFICAS}

CHANDLER, W.H., KIMBALL, M.H., PHILIP, G.L. et al Chilling requeriments for opening of buds on deciduous trees and some other plants in California. Berkeley : Calif. Agric Esp Station, 1937. 63p. (Bulletin, 611).

HELDWEIN, A.B. Disponibilidade de ocorrência de horas de frio para diferentes níveis de probabilidade na região de Santa Maria - RS. Santa Maria, 1994. 45p. Tese (Concurso para Professor Titular) - Universidade Federal de Santa Maria, 1994.

HELDWEIN, A.B., SCHNEIDER, F.M., BURIOL, G.A. $\boldsymbol{e}$ t al Disponibilidade de horas de frio na região central do Rio Grande do Sul: 1 - Ocorrência de valores acumulados para diferentes níveis de probabilidades. Ciência Rural, Santa Maria, v.30, n.5, p.747-754, 2000.

INSTITUTO DE PESQUISAS AGRONÔMICAS. Atlas climático do Rio Grande do Sul. Porto Alegre : Secretaria de Agricultura e Abastecimento, 1989. V.1. 102p.

LEDESMA, N.R. Concecuencias del frio invernal insuficiente en los arbols de follage caduco. Rev de la Fac de Agron de La Plata, La Plata, v.27, p.181-196, 1950.

MOTA, F.S. da, BEIRSDORF, M.I.C., ACOSTA, M.A.C. et al Zoneamento agroclimático do Rio Grande do Sul e Santa Catarina. Pelotas : EMBRAPA/ Secretaria da AgriculturaRS, 1974. 122p. (Circular Técnica, 5).

PETRI, J.L., PASQUAL, A. Quebra da dormência em macieira. Florianópolis : EMPASC, 1982. 54p. (Bol. Téc., 18).

Ciência Rural, v. 30, n. 5, 2000. 